\title{
A Pilot Study of an Inspection Framework for Automated Usability Guideline Reviews of Mobile Health Applications
}

\author{
Jing $\mathrm{Xu}^{1}$, Xiang Ding ${ }^{1}$, Ke Huang ${ }^{1}$, Guanling Chen ${ }^{1,2}$ \\ ${ }^{1}$ Department of Computer Science, University of Massachusetts Lowell \\ ${ }^{2}$ State Key Laboratory for Novel Software Technology, Nanjing University, China \\ \{jxu, xding, khuang, glchen\}@cs.uml.edu
}

\begin{abstract}
Usability is of significant importance for any interactive software. In the mobile domain, applications face more challenges to deliver good experiences to end users due to the characteristics and usage of mobile devices in ubiquitous computing contexts. The situation may be exacerbated for mobile health applications since the target population or domain may impose even stricter usability requirements.

Heuristic Evaluation (HE) or guideline review has proven itself to be an effective approach among many usability evaluation methods. Organizing heuristic evaluation by usability professionals, however, can be costly and time consuming, particularly for frequent prototype updates generated by fast iterations. Manual inspection by human experts also suffers from scalability issues as mobile applications often need to run on a diverse set of hardware platforms.

To help find potential usability problems at an early stage and reduce the workload of human usability experts, we propose an inspection framework to conduct automated guideline reviews of mobile health applications. The inspection framework is based on the Health Information Management Systems Society (HIMSS) usability guidelines for mHealth applications. First, we translate the high level descriptions of usability guidelines into operationalized metrics that can be measured by software. Second, we demonstrate the translation is meaningful by providing detailed analysis of suggested metrics and real-world case studies. We hope this framework can be used to enforce a minimum bar for the usability of mobile health applications and further adapted when new products in the field are developed.
\end{abstract}

\section{Categories and Subject Descriptors}

H.5.2 [User Interfaces]: Evaluation/methodology, Style Guides

\section{General Terms}

Human Factors, Verification

Permission to make digital or hard copies of all or part of this work for personal or classroom use is granted without fee provided that copies are not made or distributed for profit or commercial advantage and that copies bear this notice and the full citation on the first page. To copy otherwise, to republish, to post on servers or to redistribute to lists, requires prior specific permission and/or a fee.

Wireless Health' 14 Oct 29-31, 2014, Bethesda, MD, USA

Copyright 2014 ACM 978-1-4503-3160-9 ...\$15.00.

\section{Keywords}

Usability Testing, Guideline Reviews, Mobile Health, Mobile Applications

\section{INTRODUCTION}

As mobile devices become more pervasive, health applications are provided increasingly through mobile platforms. However, the obstacles to mobile devices attempting to provide reliable experiences for their users are numerous: the connectivity is relatively slow and unreliable; information that can be displayed on one screen is rather limited; insufficient processing capability may cause longer response times; data entry methods for mobile devices are often awkward and require a certain level of proficiency, which increases the likelihood of erroneous input especially when multiple data entry methods are used in the same application [27].

For mobile health applications, usability may be complicated since the target population of mobile applications may impose stricter requirements for use. For example, many health applications are designed to suit the needs of an older population. Many aging people, however, have never used a smartphone. Thus the mobile health application must be very easy to use. Health applications that support dosing management and drug reference in particular must not cause any misunderstanding. Such issues caused by poor usability are particularly problematic for users who suffer from low confidence regarding interactive systems and loss of visual acuity, and cognitive capacities [21].

$\mathrm{HE}$ is an effective method of providing feedback and discovering the usability problems within applications. HE involves usability experts evaluating the application based on usability guidelines and heuristics. We propose the use of operationalized usability metrics for mobile health applications and an inspection framework, which can automatically explore Graphical User Interface (GUI) of mobile health applications and identify potential usability problems. Note that, we do not intend to replace human usability experts with the inspection framework. Instead, we aim to give developers early feedback on their applications and also reduce the workload of human experts.

We envision that this automated inspection framework can be used by usability professionals to specify usability guidelines, potentially tailored for target populations (e.g. older users, young users, or physicians). Developers can then take this system to quickly check their prototypes, which often have frequent iterations due to agile programming methods. The automated system can also scale much better than manual efforts to different hardware platforms 
(e.g. Android, which supports hundreds of devices with different size, resolution, and capability). We expect that many usability problems can be caught by this inspection framework before developers run more intensive lab-based or inthe-field usability studies. This will save cost and improve productivity. In addition, a third party can use the automated inspection system to independently evaluate and rate the mobile health applications in a cost-effective way.

The contributions of this paper include: our proposed operationalized usability metrics and rules based on the analysis of HIMSS guidelines [7] for mobile health applications; our detailed analysis of the operationalized metrics that are grouped into three levels (task level, screen level, and individual widget level); our inspection framework for automated guideline reviews of mobile health applications, and the pilot study we conducted in order to evaluate the importance of suggested operationalized metrics to the usability of mobile health applications. The results of this study validated that the metrics were indeed effective and could help us prioritize the measurement implementation in our automated GUI modeling system for the proposed usability inspection framework.

Again, we do not claim an automated inspection system can capture all usability problems. Some usability guidelines cannot be easily operationalized and human experience is needed. Nor do we expect automated inspection to replace other user-centered usability design methods. Rather automated inspection aims to be an assistive toolkit that usability professionals can leverage. The quantified usability rules proposed in this paper may not be universally accepted. Usability professionals tend to have different opinions on how to implement a particular guideline. Thus the inspection system should be configurable by experts for customization to different health domains and populations. We hope this pilot study will generate discussions and feedback from the community.

\section{BACKGROUND AND RELATED WORK}

Heuristic evaluation was first developed by Rolf Molich and Jakob Nielsen [23] and subsequently refined by Jakob Nielsen [22]. It is an inspection method used to identify usability problems for interface designs. Typically, a small set of usability experts individually evaluate the user interface using a set of heuristics. Heuristic evaluation and guideline review prove to be the best methods for ensuring satisfactory design experiences [10]. They have the capability of finding the majority of usability problems of the application under evaluation [22]. However, these review methods require the usability experts to manually check the guideline conformance.

As modern applications are increasingly developed using rapid iteration methods (i.e. agile programming), heuristic evaluation is usually necessary for every prototype of each development cycle (2-4 weeks). This imposes significant time and cost overhead for small developer teams who lack usability expertise. Additionally, applications often target a diverse set of devices. Manual efforts by heuristic evaluation are insufficient at inspecting how applications work on all these devices. The behaviors may be different either intentionally (e.g. layout change due to size) or unexpectedly (i.e. due to manufacturer specific platform customization). Thus, an automated approach to heuristic evaluation and guideline review is preferable to help usability professionals cope with these challenges.

While usability heuristics and guidelines are usually expressed in natural language, the interpretation of heuristics is the most important issue for the automation. Only by transforming the usability heuristics into quantitative metrics, is it thus feasible to conduct heuristic evaluation automatically [25].

\subsection{Automated Heuristic Evaluation}

There have been a few studies on using guidelines to automatically evaluate website usability [10] [25] [11]. Vanderdonckt et al. proposed an approach to perform static analysis of HTML code against guidelines expressed in terms of Guideline Definition Language (GDL) [25]. EvalIris is a web service created to check the accessibility of web pages based on particular guidelines. The guidelines stored in EvalIris's repository can be easily updated and accommodated into the system with no significant effort by producing an XML schema of guidelines [10]. However, these technologies cannot be directly applied to the mobile platform. User interfaces of mobile applications contain much dynamically generated content. This content cannot be easily examined through static analysis as it is not described using standard language (such as HTML). In addition, due to the small screen size and limited processing capability, the user interfaces of mobile application are different than websites and need to follow unique usability guidelines.

\subsection{Guidelines for Mobile Health Applications}

To automate heuristic evaluation of mobile health applications, the first step is to identify heuristic guidelines in health domain for mobile devices. Nielsen's 10 usability heuristics set has been the most widely used since 1994. Nielsen's 10 established the cornerstone of heuristic evaluation. Mobile devices and wireless technologies have provided richer user experiences with more media-rich and context-aware features. Specialized heuristics were developed for specific devices, applications, or targeted users [16] [18] [13]. There are several studies on accessibility and usability testing of mobile devices in the health domain [19] [14]. However, the majority of previous mobile device usability studies have focused on the use of PDAs and applications on mobile devices not supporting multi-touch interactions, while a lot of applications now target a much wider scope [4].

Guidelines geared towards mobile health applications include those published by Happtique Health App Certification Program (HACP), the Food and Drug Administration (FDA), and Health Information Management Systems Society (HIMSS). The guidelines provided by HACP assist healthcare providers and consumers identify medical, health, and fitness applications that "deliver credible content, contain safeguards for user data, and function as described" [2]. The HACP guidelines mainly focus on the integrity, privacy, and security of data by providing a detailed list of mobile health application standards. The FDA has also published guidelines for medical applications. They do not, however, focus on usability [5], either. The FDA guidelines attempt to define and regulate the boundary between mobile applications and mobile medical applications.

The HIMSS guidelines are the first comprehensive set of usability heuristics developed for mobile health applications. They are an augmented version of Nielson's 10 usability heuristics. Moreover, these guidelines take into account not 
only mobile application usability, but also important principles of health applications. Listed below are the 9 HIMSS guidelines for mobile health applications. Note that HIMSS provides a detailed explanation of those guidelines. We have listed the simplified version in the interest of saving space.

- Simplicity

- Naturalness

- Consistency

- Forgiveness and feedback

- Effective use of language

- Efficient interactions

- Effective information presentation

- Preservation of context

- Minimum cognitive overload

The HIMSS guidelines need to be operationalized for automated inspection. The work of Automated Model checker (AMC) [20] evaluated 7 user interface properties of mobile vehicle applications. These properties are: user actions per task; text contrast ratio; text word count; button size; button distance; animation and scrolling. These properties are based on two key principles of user interface guidelines for vehicular applications: "minimum distraction" and "minimum memory load". However, mobile health applications do not have a requirement for "not disturbing users" as vehicle applications. To operationalize the HIMSS guidelines for mobile health applications, we outline all measurable metrics in the proposed inspection framework in Section 3 of this paper. We categorize them into three different levels: task level, screen level, and individual widget level. The actual threshold for those quantitative metrics can be customized depending on usability expert's experience or the needs of different populations. Currently, we design threshold to be configurable in the proposed inspection framework for mobile health application.

\subsection{Coarse Grained GUI Model (CGGM) of Mobile Applications}

Before we look at the details of the operationalized guidelines, we should first introduce key concepts of our Coarse Grained GUI Model (CGGM) on which the inspection framework is based [26]. In the CGGM, we describe mobile applications using state machines. Each state represents a unique screen of the application. To avoid state explosion and allow regressive modeling, we use structure as the unique feature to define the equivalence of states. The structure of a screen is represented by its set of widget groups. The widgets inside one group are homogeneous and defined as siblings. The most important property of a group is homogeneity.

In essence, our inspection framework, currently supported on Android platform, can run target application in emulator or on actual devices to systematically explore the application's view and layout properties and screen transition models. This process is automated without user intervention. The application information obtained can be compared against the operationalized usability heuristic/guidelines as described below.

\section{OPERATIONALIZED METRICS}

In this section, we will provide a detailed analysis of the proposed metrics, categorize the metrics into three different hierarchical groups, and explain how they could be executed as basic usability standards for mobile health applications. Our metrics have not been randomly selected. Rather, each metric corresponds to important usability elements in the HIMSS guideline. For example, metric "Number of steps to complete a task" corresponds to whether the application follows "Simplicity" and "Minimum cognitive overload" guidelines.

\subsection{Task Level Metrics}

A task is defined as a sequence of application interaction steps that achieve a certain objective. In the context of CGGM, a task is a state-transition path from the starting state, to some other state(s), and then back to the starting state [20]. While some tasks derived from this approach may not be meaningful, it does give us a way to systematically explore all meaningful tasks without any developer input. We discuss how this can be implemented in CGGM in the next section.

Number of steps to complete a task. The user's cognitive load increases when more steps are required to complete a task. This may decrease task completion especially for applications targeting older users. It is advantageous for the tasks to be as simple as possible.

Number of data inputs required to complete a task. Asking for data input excessively disturbs user from perceiving the information content of the interface as the human working memory has to repeatedly switch between entering data and understanding meaning. This becomes particularly challenging as virtual keyboards cover large portions of the screen.

Pattern of action sequences for completing tasks. To design a consistent interface, understanding how one screen works helps users understand how other screens work [7]. If the tasks follow a similar workflow, it is easier for users to learn how to use the application.

If the operation requires more response time, does it provide any explanatory information? Since mobile applications are mostly event-driven, the UI process is separated from the computation processes behind it. However, mobile devices have limited computing capacity and may have slow network connections. When there is a noticeable delay in UI processing, the user should receive explanatory feedback. Otherwise, users could easily think the interface has crashed or is frozen.

Number of operations that require more system resources. If the triggered action demands more system resources, it is likely the application requires a longer response time. Excessive waiting exhausts a user's patience and disturbs their workflow.

\subsection{Screen Level Metrics}

Percentage of screen space used and number of text of visible widgets. Visible widgets and the text on widgets belong to the information content (IC) of the screen. IC correlates to the potential for conveying meaning to users. According to the equation: Display Complexity (DC) $=$ Information Content (IC) + Layout Content (LC) + Visual Noise (VN), the complexity of the interface increases as the number of visible widgets and text count increases [15]. 
Are the widgets well organized? There should be no overlapping of groups. In our inspection framework, a group must enclose all homogeneous widgets. Groups should divide the screen into different parts in terms of functions. To make the screen simple and easy to learn, no groups should overlap. Namely, the functional areas need to be easily distinguished.

Average operation distance on the screen. The distance between two operations on the screen is defined as $d=\sqrt{\left(x_{1}-x_{2}\right)^{2}+\left(y_{1}-y_{2}\right)^{2}}$, where $\left(x_{i}, y_{i}\right)$ is the location of the touch point of the operation. Users take longer to complete tasks with large operation distances. The operation distance can be decreased by providing a more appropriate layout and demanding fewer operations from users $[24]$.

Is the layout organized consistently? Are margins, font, captions consistent? When users understand a single screen, they are more likely to understand additional screens in the same application. Therefore, consistence of layouts is critical. Consistency helps reduce the cognitive load of users and accelerates their completion of the task.

Number of medical terms. Are there any jargon in the text? More than half of American adults have low health literacy. Those with lower educational status have even less literate regarding their health [12]. To be effective, the application must use everyday language. Since most health applications target users with multiple health literacy levels, the health application should use little medical terms as possible. There should be no jargon in the text.

Number of ListView item count and MenuItem count. When facing many choices, users have more difficulty making the right decision. People tend to feel tired after browsing certain choices and usually stop at one that may not necessarily lead to a satisfactory result.

Number of scrollable groups. Scrolling through many options kills a user's patience, especially when interacting on a small screen. If all navigation options are visible and users do not have to scroll to navigate, users can then rely on recognition rather than recall [6].

\subsection{Individual Widget Level Metrics}

For widgets leading to state transitions, is it in a group of size 1? How much space does it occupy? One important factor for ensuring the simplicity of mobile applications is that "important information stands out and function options are easy to understand" [7]. A group on the screen is a partition of functions. User events that lead to state transitions are usually registered with important functions, such as clicking the "Log In" button on the screen. To highlight such widgets, one possible solution is to distinguish the widget as a unique function group of size 1 . Another possiblity is to attract user's attention with more space on the screen and use an image rather than text.

Does the text input field have default value and support one-click delete? Default value and one-click delete help improve the efficiency of data input and editing. These efficiencies are consistent and expected in most current mobile applications.

Font, size, and color contrast ratio of text. Appropriate font should be adopted consistently among different screens to reduce the abruptness when users move through the application. For applications targeting at older populations who may suffer from loss of visual acuity, larger size and appropriate contrast ratio of text is recommended [17].

No inappropriate usage of red color. Colors convey meaning to users in the context of medical applications, red usually indicates urgency/emergency. The inappropriate use of the color red could cause unnecessary agitation and anxiety in users [7]. A color coding system has been developed to reduce confusion and support decision making. Warning information is displayed in red. Caution information is displayed in yellow or amber. Advisory information is displayed in another color [1].

\section{MEASUREMENTS FOR AUTOMATED IN- SPECTION}

In this section, we introduce how the proposed metrics can be measured automatically. The inspection framework is based on our Coarse Grained GUI Model (CGGM) tool. This tool can automatically model GUIs of mobile applications through iterative exploration [26]. This tool uses the structure information to summarize interfaces on the screen, which enables regressive modeling and merging of GUI models under different contexts. The CGGM modeling tool produces a state machine model of the mobile application. Each state represents screen views that have the same structure summary. The transitions between states are invoked by GUI events triggered on the screen, such as a tapping event. The structure of a screen view is defined by the sets of groups that constitute the screen view. All widgets in one group share the same behaviors. Thus groups are essentially the partition of widgets on the screen in terms of functionality.

We next describe how measurements can be collected from CGGM models and then used to calculate the metrics described in Section 3. We hope usability professionals can configure the inspection framework for the thresholds of these metrics and tailor these thresholds for different health domains and populations.

\subsection{Task Level Measurement}

Task complexity measurement. The prior model checker defines a task as the shortest path from one state to another and back [20]. However, we found that this definition is inadequate in regards to round-trip paths of length 2 or length 3, which have no real meaning. This definition also neglects important tasks for users with different experiences. As shown in Figure 1, path ABCDA may be an important path adopted by a novice user. The application may, however, also provide a shortcut such as a "customized gesture" for experienced users through path ADA. Because path ADA has a shorter length, path ABCDA would not be identified as a task by the definition.

In our inspection framework, we allow developers to configure the lower limit (denoted as $m$ ) of the task length. A task is defined as the shortest path from state $A$ to state $\mathbf{B}$ back with at least $m$ states (other than A, B) included in the route. For each value of $m$, we have a task set to include all the paths that satisfy the definition. As show in Figure 1, for tasks starting from state A, we have:

- when $m=2$, that task set is $\{\mathrm{ABCDA}\}$.

- when $m=1$, the task set is ABDA, ACDA, ABCA, ADCA, ABCDA\}. 
- when $m=0$, the task set is ABA, ADA, ACA, ABDA, ACDA, ABCA, ADCA, ABCDA .

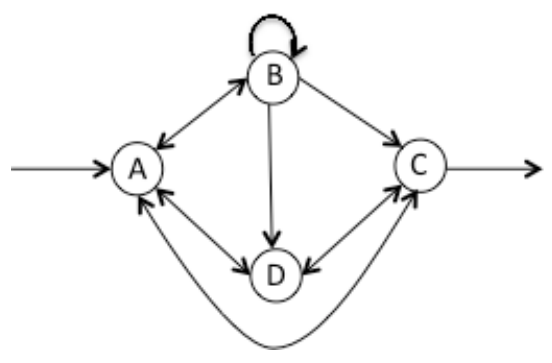

Figure 1: Task pathes

Note that the task set with $m=i+1$ is a subset of the task set with $m=i$. When the task length exceeds an upper limit, tasks are marked as violations.

Data inputs measurement. Data entering is different from data input controls as described in the Android document. In our inspection framwork, data entering refers to a user entering text through typing or voice control. Data input controls include all of the interactive components on the interface, such as buttons. We do not consider swiping and tapping to be data entering as they do not cause much distraction. Thus, only operations on widgets that are subclasses of EditText are counted as data entering.

Operation pattern measurement. Tasks in the CGGM can be translated into strings using a code table. Each character in the code represents a single operation. The longest common substring algorithm can detect patterns repeated among all of the strings. The detected patterns can then be decoded according to the previously used code table. To prevent the detection of any pattern between a task and all its subtasks, we do not apply this algorithm to tasks with different lengths. We also do not compare tasks with the same starting state.

Response time measurement. When beginning a new transition, the CGGM tool pauses for 2 seconds to capture the new screen view. It first examines whether any widget of ProgressBar type exists. Once such a widgets is detected, the operation is marked and more response time is required. The CGGM tool captures the screen view at an interval of every two seconds until the widget of ProgressBar type disappears. Under certain conditions, the CGGM tool would not move on. Instead, it sets a timeout of 5 minutes if the screen view gets stuck. In that case, a crash will be reported and the exploring process will end. As the CGGM tool extracts the GUI model by multiple runs, it would exclude all previously discovered crash points.

\subsection{Screen Level Measurement}

The inspection framework first determines the height and width of a pixel on the screen. The framwork then uses the number of pixels to calculate the size and location of different user interface elements on the screen. The location $(x, y)$ of an interface element is computed in the coordinate system where the origin sits at the leftmost and uppermost point of the screen. The CGGM tool will extract the size and position of visible widgets during the exploration of the application.

Space usage and layout properties measurement. Android allows custom views to be added to the application.
Width and height properties of irregular interface elements are sometimes not available. One possible solution is to count the number of pixels the view occupies. For simplicity's sake, in the case width and height properties are not available, we calculate the size of the custom view's direct container instead.

To check the consistency of the layout of the application, the inspection framework examines whether margins, background colors, and the first widget group on the screen views are consistent. According to the exploration algorithm of the CGGM tool, the first widget groups on the screen view are usually the signature icon or label of the application.

Operation distance measurement. The operation distance between two operations on the screen is defined as $d=\sqrt{\left(x_{1}-x_{2}\right)^{2}+\left(y_{1}-y_{2}\right)^{2}}$. The operation distance measured by the inspection framework is the estimation of finger movement on a touch screen. While users are not involved, the inspection framework uses the location of the widget's center point $\left(x_{i}, y_{i}\right)$ as the coordinate of touch point. For custom views, it measures the location of direct container's center point.

Medical terms. Many medical dictionaries are available online [3]. The inspection framework processes extracted text word by word and compares the text against terminologies listed in the dictionary. When finding a match of one term, the inspection framework adds 1 to the count of medical terms.

\subsection{Individual Widget Level Measurement}

Widget properties measurement. The most appropriate fonts, sizes, and colors for mobile health applications are not well established. User interfaces should adapt to different resolutions and sizes on various mobile devices for the same application. The CGGM tool can already identify widget properties such as size, location, text, and whether widgets are scrollable, clickable, and etc. The inspection framework can also extract color information using third-party tools to compute the color contrast ratio of texts [9]. Text on images can be handled with Optical Character Recognition (OCR). 3rd party OCR tools already exist[8].

\section{CASE STUDIES}

We selected 24 mobile health applications from the Google Play store for case studies. To make meaningful comparisons between selected applications, all applications are diabetes management tools. We searched the Google Play market with keyword "diabetes" from free applications and the returned applications are ranked in order. We chose the applications with the highest ranking. For better reference, we list the selected applications below.

The purpose of our case studies is to answer the following questions:

- Diabetes management is an important issue. Are the highly rated applications of good quality from the perspective of usability? Can our operationalized metrics help find major usability problems? Are they effective?

- We have not established any specific value/threshold for quantitative metrics. Is an upper bound or lower bound always good?

The selected applications were evaluated by a human expert against the suggested metrics. Metrics related to a usability problem are marked. The human expert also recorded 


\begin{tabular}{|l|c|}
\hline Name & Rating/Downloads \\
\hline Diabetic's Logbook & $4.2 / 5,000+$ \\
\hline Diabetes Journal (MACDEV) & $4.5 / 1,000+$ \\
\hline Diabetes Tracker & $3.8 / 10,000+$ \\
\hline Diabetes Journal & $4.5 / 50,000+$ \\
\hline Diabetes Plus & $4.3 / 10,000+$ \\
\hline OnTrack Diabetes & $4.4 / 500,000+$ \\
\hline Diabetes Diary Lite & $4.1 / 10,000+$ \\
\hline Diabetes - Glucose Diary & $4.2 / 100,000+$ \\
\hline Glucose Buddy: Diabetes Log & $4.4 / 100,000+$ \\
\hline My Diabetes & $4.6 / 10,000+$ \\
\hline Diabetes Connect & $4.5 / 10,000+$ \\
\hline Diabetes Tracker (MIG SUPER) & $4.0 / 10,000+$ \\
\hline Social Diabetes & $4.4 / 10,000+$ \\
\hline Diabetes Diary & $4.0 / 10,000+$ \\
\hline BG Monitor Diabetes & $4.6 / 10,000+$ \\
\hline Glucool Diabetes & $3.6 / 10,000+$ \\
\hline SiDiary Diabetes Management & $4.4 / 50,000+$ \\
\hline DMcontrol - Diabetes & $4.3 / 1,000+$ \\
\hline Diabetes & $4.4 / 1,000+$ \\
\hline Diabetes Vue & $4.5 / 1,000+$ \\
\hline Diabetes Diary Glucose Tracker & $3.8 / 5,000+$ \\
\hline Diabetes Log & $4.3 / 1,000+$ \\
\hline Diabetes Log Book & $3.6 / 10,000+$ \\
\hline Diabetes Tools & $4.5 / 1,000+$ \\
\hline
\end{tabular}

Table 1: Apps for Evaluation

usability problems that could not be revealed by examining the suggested metrics.

\subsection{Evaluation Results}

All 24 were found to have usability problems according to the suggested metrics. No usability problem was found related to these metrics: consistency of fonts and colors used, number of medical terminologies, inappropriate use of the color red. The human expert discovered usability problems for all other metrics.

Metrics with the most usability problems found are: number of steps to complete a task, number of scrolling operations required, appropriate font, number of data entering required, color contrast, text count, and screen space usage.

Below are the 11 most severe and common usability problems of the 24 highly rated applications as follows. Table 1 summarizes the results.

1. The application only provides one simple function, though it does not violate any guidelines (Such as "Diabetes Diary").

2. There is too much text and too many numbers on the screen. Consequently the text and numbers are too small to read.

3. There is no default value of data entries for text inputs.

4. The application asks users to type a date instead of selecting a date from a picker.

5. The navigation does not intuit that users would be brought back to a starting point unless they hit the "Back" button. (Usually when a task is finished, the application would bring users to the initial interface.)

\begin{tabular}{|c|c|c|c|}
\hline $\begin{array}{c}\text { Usability } \\
\text { Problem \# }\end{array}$ & $\begin{array}{c}\text { Metric } \\
\text { Level }\end{array}$ & $\begin{array}{c}\text { Number of Apps } \\
\text { Found with the } \\
\text { Problem }\end{array}$ & $\begin{array}{l}\text { Average } \\
\text { Rating }\end{array}$ \\
\hline 1 & Task & 6 & 4.0 \\
\hline 2 & Screen & 8 & 4.3 \\
\hline 3 & Widget & 1 & 4.5 \\
\hline 4 & Widget & 1 & 4.5 \\
\hline 5 & Task & 3 & 3.9 \\
\hline 6 & Screen & 8 & 4.2 \\
\hline 7 & Screen & 11 & 4.2 \\
\hline 8 & Screen & 7 & 4.3 \\
\hline 9 & Screen & 2 & 4.2 \\
\hline 10 & Screen & 1 & 4.2 \\
\hline 11 & Widget & 3 & 4.1 \\
\hline
\end{tabular}

Table 2: Major usability problems found of the 24 diabetes logging applications.

6. Too much data entering is required in one single screen view.

7. The list is so long that users have to scroll several times to select an item from the bottom of the list.

8. The color contrast is not appropriate.

9. The application uses too many colors in one screen view.

10. The layout is not consistent with other applications. Most applications are adopting the left-aligned layout, while the application uses a center-aligned layout.

11. Interactive widgets do not stand out. They only occupy a small percentage of screen space.

\subsection{Discussions}

The selected applications have an average rating score greater than 4.2 on Google play. We consider user reviews for an application important indicator of usability. The usability problems we detected are consistent with user reviews.

We see developers have already paid attention to some important metrics, which are universally good for all 24 applicaions. We didn't find any usability problem regarding these metrics: consistency of fonts and colors used, number of medical terminologies used, inappropriate use of the color red. Other metrics, however, are not yet fully considered. This does not mean the metrics that didn't find usability problem with are not helpful. On the contrary, these developments show that those metrics have already been adopted as minimum standards for usability of mobile health applications. As well, even though those applications have a relatively higher rating score, we can still find severe usability problems by checking them against our operationalized metrics.

Based on these results, we can answer the first question saying the suggested metrics are important ingredients to the usability of mobile health applications. They are indeed effective in locating major usability problems through automated inspection. We do not claim those quantitative metrics can capture all usability problems, as some usability guidelines can not be easily operationalized. Instead, 
our suggested metrics identify the minimum usability standards for mobile health applications. We hope our metrics can help provide early usability feedbacks for products and reduce workload of human usability experts.

Regarding the second question, we can say that metrics such as text count, total colors used, space usage of interactive widgets, amount of scrollings, data inputs on one screen and data inputs throughout the task, our study proved that an upper bound is necessary for these metrics. One phenomenon of interest to us is the commonly held assumption that the fewer the number of steps, the better the application. The severe usability problem related to this metric is that some applications provide only a very simple function though they do not violate any other guidelines. We reviewed applications having such a problem and found that those applications have a relatively lower rating among all 24 apps. They have a lot of tasks of short length (no more than 2) and do not have long-sequence tasks. Though we argued in Section 3 that tasks with long length would test a user's patience and increase their cognitive load, applications with only short tasks should also be further examined since they might be too simple to compete on the market. The metrics such as "number of steps of a task" needs to be further studied to see whether there is a good threshold value in terms of usability of mobile health applications. Currently, we do not suggest a universal threshold for any quatitative metric. The thresholds themselves should also be adapted as new types of applications are developed in the field. The next step is to run well-known applications in our inspection framework and study whether there could be a general threshold for each metric.

\section{CONCLUSION}

In this paper, we proposed an inspection framework for automated usability guideline reviews of mobile health applications. The most significant contribution of our work is that we translated the high-level language description of usability guidelines (HIMSS) into quantitative metrics that could be automatically measured by software. The main purpose of this work is to demonstrate our translation is meaningful in two ways: 1) we explained in detail in Section 3 how each operationalized metric can correspond to important usability elements; 2) we also demonstrated that severe usability problems can be found by checking them against proposed metrics. The next step is to develop the fully automated framework. However it is not conceivable that an automated inspection framework could capture all usability problems, nor could it replace human usability experts. We hope that the proposed system can be used to set a minimum bar for the usability of mobile health applications and the field in general can benefit from our work.

\section{Acknowledgment}

This work is supported partly by the National Science Foundation under Grant No. 1016823 and No. 1040725. Any opinions, findings, and conclusions or recommendations expressed in this work are those of the author(s) and do not necessarily reflect the views of the National Science Foundation. We would also like to thank Wendy Nilsen for shepherding this paper.

\section{REFERENCES}

[1] The application of color in healthcare settings, http://www.ki.com/.

[2] Health app certification program, http://www.happtique.com/.

[3] http://www.medilexicon.com.

[4] Mobile medical and health apps: state of the art, concerns, regulatory control and certification, online journal of public health informatics, http://ojphi.org.

[5] Mobile medical applications - guidance for industry and food and drug administration staff, http://www.fda.gov.

[6] Mobile navigation: Image grids or text lists?, http://www.nngroup.com.

[7] Selecting a mobile app: Evaluating the usability of medical applications, http://www.himss.org.

[8] Tesseract-ocr, https://code.google.com/p/tesseract-ocr/.

[9] Web content accessibility guidelines, http://www.w3.org/tr/understanding-wcag20.

[10] J. Abascal, M. Arrue, I. Fajardo, N. Garay, and J. Tomás. The use of guidelines to automatically verify web accessibility. Universal Access in the Information Society, 3(1):71-79, 2004.

[11] T. Brinck and E. Hofer. Automatically evaluating the usability of web sites. In CHI '02 Extended Abstracts on Human Factors in Computing Systems, CHI EA '02, pages 906-907, New York, NY, USA, 2002. ACM.

[12] J. Broderick, T. Devine, E. Langhans, A. J. Lemerise, S. Lier, and L. Harris. Designing health literate mobile apps. 2014.

[13] A. Holzinger, G. Searle, and A. Nischelwitzer. On some aspects of improving mobile applications for the elderly. In Universal Acess in Human Computer Interaction. Coping with Diversity, volume 4554, pages 923-932. Springer Berlin Heidelberg, 2007.

[14] R. Hubert. Accessibility and usability guidelines for mobile devices in home health monitoring. SIGACCESS Access. Comput., (84):26-29, Jan. 2006.

[15] J. Hugo and D. Gertman. A qualitative method to estimate hsi display complexity. Nuclear Engineering and Technology, 45(2):141-150, 2013.

[16] R. Inostroza, C. Rusu, S. Roncagliolo, C. Jimenez, and V. Rusu. Usability heuristics for touchscreen-based mobile devices. In Information Technology: New Generations (ITNG), 2012 Ninth International Conference on, pages 662-667, April 2012.

[17] S. Kim, A. K. Dey, J. Lee, and J. Forlizzi. Usability of car dashboard displays for elder drivers. In Proceedings of the SIGCHI Conference on Human Factors in Computing Systems, CHI '11, pages 493-502, New York, NY, USA, 2011. ACM.

[18] H. Korhonen and E. M. I. Koivisto. Playability heuristics for mobile games. In Proceedings of the 8th Conference on Human-computer Interaction with Mobile Devices and Services, MobileHCI '06, pages 9-16, New York, NY, USA, 2006. ACM.

[19] S. Kundu, J. Mukherjee, A. K. Majumdar, B. Majumdar, and S. Sekhar Ray. Algorithms and heuristics for efficient medical information display in pda. Comput. Biol. Med., 37(9):1272-1282, Sept. 2007.

[20] K. Lee, J. Flinn, T. Giuli, B. Noble, and C. Peplin. 
Amc: Verifying user interface properties for vehicular applications. In Proceeding of the 11th Annual

International Conference on Mobile Systems, Applications, and Services, MobiSys '13, pages 1-12, New York, NY, USA, 2013. ACM.

[21] A. Lorenz and R. Oppermann. Mobile health monitoring for the elderly: Designing for diversity. Pervasive and Mobile Computing, 5(5):478 - 495, 2009.

[22] J. Nielsen. Enhancing the explanatory power of usability heuristics. In Proceedings of the SIGCHI Conference on Human Factors in Computing Systems, CHI '94, pages 152-158, New York, NY, USA, 1994. ACM.

[23] J. Nielsen and R. Molich. Heuristic evaluation of user interfaces. In Proceedings of the SIGCHI Conference on Human Factors in Computing Systems, CHI '90, pages 249-256, New York, NY, USA, 1990. ACM.

[24] H. Okada and T. Asahi. Guitester: A log-based usability testing tool for graphical user interfaces. IEICE Transactions on information and systems, 82(6):1030-1041, 1999.

[25] J. Vanderdonckt, A. Beirekdar, and M. Noirhomme-Fraiture. Automated evaluation of web usability and accessibility by guideline review. In $W e b$ Engineering, volume 3140 of Lecture Notes in Computer Science, pages 17-30. Springer Berlin Heidelberg, 2004.

[26] J. Xu, X. Ding, G. Chen, J. Drury, L. Wang, and X. Li. A new method for automated gui modeling of mobile applications. In Mobile and Ubiquitous Systems: Networking Services, MobiQuitous, 2013. MobiQuitous '13. 10th Annual International. IEEE, December 2013.

[27] D. Zhang and B. Adipat. Challenges, methodologies, and issues in the usability testing of mobile applications. International Journal of Human-Computer Interaction, 18(3):293-308, 2005. 


\section{Note:}

We revised the paper based on answers to reviewers' comments as follows to improve the paper.

- Need more work in grammar and layout and the results of case studies should be better explained.

We fixed grammar and layout issues and made it clear. We reviewed the results and explain in more details.

- Grammar issues and needs to be more succinct.

We found a native English speaker to help proofreading and editing.

- Why manually assess?

We proposed an automated usability-testing framework for mobile health applications based on our previous work in automated modeling of mobile applications. The most significant contribution is that the suggested framework actually translates the usability guidelines (in high-level language) to quantitative metrics that can be measured by software. The main purpose of this paper is to demonstrate the translation is correct in both ways: 1 ) we explained in details why each guideline could be translated into corresponding metrics in Section $3 ; 2$ ) we also demonstrated that severe usability problems can be found by examining against those suggested metrics. The next step is to develop the fully automated framework. We will articulate this point better.

- Compare evaluation with some actual usability feedback from consumers.

We consider user reviews for an app as an important indicator of usability feedback from consumers. The usability problems detected are actually consistent with user reviews.

- The HIMSS guidelines are not limited to health applicationspecific issues - they are quite general and low level.

As we addressed in the paper, we do not claim an automated inspection system can capture all usability problems, as some usability guidelines cannot be easily operationalized (human experience is needed), nor do we expect it to replace other usability evaluation methods. It aims to be an assistive toolkit that usability professionals can leverage.

- The paper didn't discuss what the work reveals about specific issues related to diabetes management. There is no testing with patients or users more generally.

The most significant contribution is that the suggested framework actually translates the usability guidelines (in high-level language) to quantitative metrics that can be measured by software. The main purpose of this paper is to demonstrate the translation is correct in both ways. We do not intend to discuss specific issues related to diabetes managements. We chose diabetes management apps for case studies for the following reasons: 1) Diabetes management is an extremely important health issue nowadays. We want to see whether those diabetes management apps are of good usability or not. 2) There are a lot of Diabetes management apps available from Google Play. It might be difficult for us to find these many apps in other health domain.

- The operationalized metrics are very qualitative. Who decided what? PLEASE provide more methodological support on a) how the metrics have been derived b) how they would be evaluated as a gold standard (expert involvement?) c) how much error is introduced because of automation of the tests? What would be a gold standard (expert opinion? user study?) to compare to and evaluate if the proposed framework is accurate? Why this step has not been taken? How is the evidence handled in the framework? Case studies: what were the apps? free? paid? how many users? Having an app listed by google play doesn't say anything and should not be taken as a reason for inclusion in the scientific studies. Have you contacted the developers of the apps informing them about your results? Ideally you would let an expert user evaluate the apps vs. compare to your framework assessing automatically the apps.

There might be some misunderstandings of this paper for this comment. We explained in details in Section 3 how each HIMSS guidelines were translated into measurable metrics and why those metrics can reflect the important usability aspects of its corresponding guideline.

We never claim the proposed framework should be considered as golden standards. We noted very clearly in the last paragraph of Section 1 that "We do not claim an automated inspection system can capture all usability problems, as some usability guidelines cannot be easily operationalized (human experience is needed). Nor do we expect it to replace other user-centered usability design methods. Rather it aims to be an assistive toolkit hat usability professionals can leverage. The quantified usability rules proposed in this paper may not be universally accepted, as usability professionals may have different opinions on how to implement a particular guideline. Thus the inspection system should be configured by experts for customization to different health domains and populations. We hope this pilot study will generate discussions and feedback from the community."

Also, HIMSS guidelines is the most relevant usability guidelines specialized for mobile health apps The suggested metrics are based on HIMSS guides and can address most important issues in mobile usability. We demonstrated in case studies that severe usability problems were detected by evaluating against those metrics.

The reason why we chose diabetes management apps for case studies is that diabetes management is an extremely important health issue nowadays. We also want to see whether those diabetes management apps are of good usability or not. The selected apps are the most highly rated diabetes management apps from Google Play, which is the official platform for developers to deliver their apps on Android platform.

The purpose of the case studies is to demonstrate the 
effectiveness of the propose framework in finding usability issues of mobile health apps. As we noted in the paper, the proposed framework is not to replace human usability experts and it cannot capture all usability problems. It is aimed to be a toolkit to reduce the workload of human usability expert and help developers find usability problems at an early stage. We plan to include a table listing the apps under study.

- The paper needs to highlight the need for this to health. They also need to highlight how this would be the minimum usability standard for mHealth. It would only set a baseline, which would be adapted as new products are developed.

We intentionally selected the most highly rated diabetes management apps and want to see if there are still severe usability issues even though they have a relatively higher rating score. According to the result of our case studies, major usability problems were still discovered. Thus, there is an urgent necessity to improve the usability of mobile health apps.

As we stated in the paper, the HIMSS guidelines were developed from Nielsen Usability Heuristics, which has been the cornerstone of heuristic usability evaluation and the baseline for all software products. We articulated this point more clearly in the paper. 